\title{
An atypical manifestation of primary cardiac tumor in a young patient
}

\author{
Michat Chyrchel ${ }^{1}$, Bernadeta Chyrchel ${ }^{1}$, Artur Dziewierz ${ }^{1}$, Agnieszka Rożanowska ${ }^{2}$, Ewa Czestkowska ${ }^{2}$, \\ Andrzej Surdacki ${ }^{1}$, Dariusz Dudek ${ }^{1}$ \\ ${ }^{1} 2^{\text {nd }}$ Department of Cardiology, Jagiellonian University Medical College, Krakow, Poland \\ ${ }^{2}$ Students' Scientific Group at the $2^{\text {nd }}$ Department of Cardiology, Jagiellonian University Medical College, Krakow, Poland
}

Adv Interv Cardiol 2020; 16, 1 (59): 110-111

DOI: https://doi.org/10.5114/aic.2019.91300

A 31-year-old male patient was admitted to a tertiary hospital due to chest pain, dyspnea, fever, and cough lasting a week before admission. The patient reported a respiratory tract infection a month earlier. On admission an elevated level of leukocytes up to $6.80 \times 10^{9} / \mathrm{l}$ and C-reactive protein up to $105.2 \mathrm{mg} / \mathrm{l}$ were found. On chest $\mathrm{X}$-ray enlargement of the heart was observed with no abnormalities in ECG (Figure $1 \mathrm{~A}$ ). As in the echocardiography a significant amount of fluid was found in the pericardium an initial diagnosis of pericarditis was established. The patient was successfully treated pharmacologically and after nine days was discharged home in good condition. He was scheduled for rheumatology consultation. After 2 weeks, in control echocardiography no abnormalities were found. However, after 3.5 months, he was admitted again due to severe dyspnea and atypical chest pain. On X-ray, the presence of many circular lesions in both lungs was confirmed. Also, in the echocardiogram a large tumor in the right heart ventricle was observed (Figure $1 \mathrm{~B}$ ). Chest computed tomography (CT) confirmed the presence of the tumor of the right heart and metastatic lesions in both lungs (Figure $1 \mathrm{C}$ ). The patient was transferred to the reference center for further assessment and treatment. Control echocardiography, $\mathrm{CT}$, cardiac magnetic resonance (CMR) as well as positron emission tomography (PET) scans were performed to confirm expansion of the tumor within the heart walls (Figures 1 D, E). Coronarography was done to visualize potential connections between coronary arteries and tumor vessels (Figure 1 F). The myocardial biopsy confirmed the presence of angiosarcoma of the heart. The patient was discussed by the Heart Team and, at that stage, was disqualified from surgery. He was scheduled for palliative chemotherapy with doxorubicin $\left(75 \mathrm{mg} / \mathrm{m}^{2}\right)$. Echocardiographic follow-up was performed every 3 weeks before each cycle of chemotherapy, but only a small reduction in the size of the tumor was observed (Figure $1 \mathrm{G}$ ). Due to the limited response to chemotherapy, the patient was disqualified from surgery again. In the control CT scan, progression of tumor growth with numerous metastatic lesions in the lungs was observed (Figure $1 \mathrm{H}$ ). Gradual worsening of symptoms was observed, and the patient died 7 months after the diagnosis.

Primary heart tumors are relatively rare and only $25 \%$ of heart tumors are malignant (mostly represented by angiosarcoma) [1]. Heart angiosarcoma more often affects young patients, especially men [1]. Most frequently, angiosarcoma of the heart occurs in the right atrium with a tendency to infiltrate surrounding tissues (pericardium, large vessels). The localization of angiosarcoma within the right ventricle is exceptional (about $4 \%$ of cases). Due to its primary localization, clinical symptoms at the beginning are not characteristic. For instance, the first manifestation of the heart angiosarcoma could be pericardial effusion, as in our case [2]. However, in the case of pericardial effusion, further diagnostics more frequently lead to the confirmation of myocarditis, rheumatologic diseases, or collagenases, especially in young patients. More noticeable symptoms such as easy fatigue, dyspnea, and right-sided cardiac failure are usually associated with the dynamic growth of the tumor mass [1]. Apart from echocardiography, CT, CMR, and PET are routinely performed for precise visualization, tumor infiltration of local structures, and possible metastatic lesions [3, 4]. Imaging studies are crucial in cases scheduled for cardiac surgery. Possible therapeutic options include cardiac surgery, radiotherapy, or aggressive chemotherapy. However, in most cases, the prognosis is poor, with a median survival of less than 11 months after the diagnosis [5].

\section{Corresponding author:}

Michal Chyrchel MD, $2^{\text {nd }}$ Department of Cardiology, Jagiellonian University Medical College, 15 Kopernika St, 31-501 Krakow, Poland, phone: +48 502595 154, e-mail: mchyrchel@su.krakow.pl

Received: 14.08.2019, accepted: 15.08.2019. 

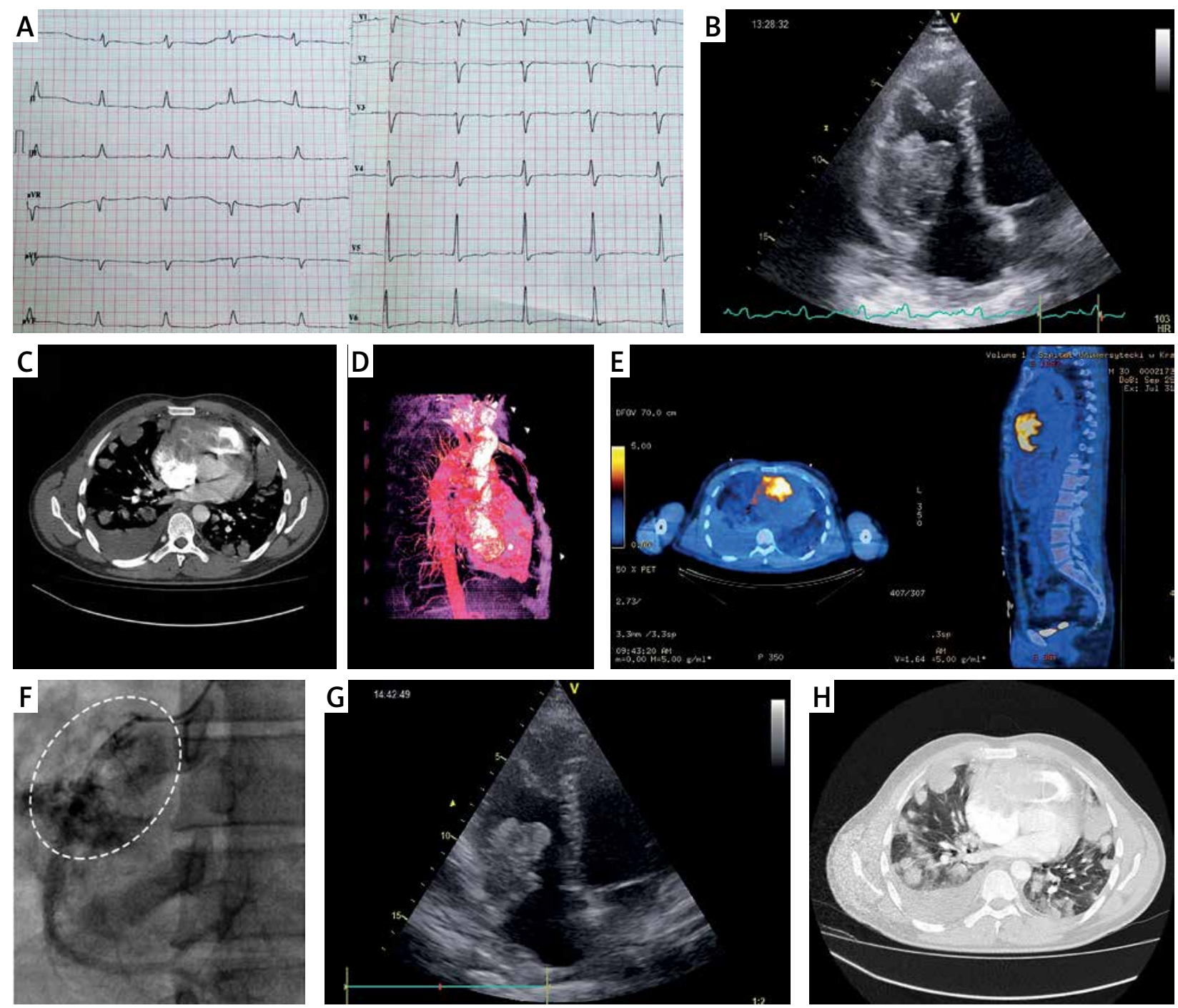

Figure 1. Heart angiosarcoma in the 31-year-old man. A - initial electrocardiogram without any significant abnormalities, $\mathbf{B}$ - echocardiogram with a large tumor in the right heart ventricle, $\mathbf{C}$ - chest computed tomography $(C T)$ with the presence of the tumor of the right heart and metastatic lesions in both lungs, D, E - CT and positron emission tomography (PET) scans, $\mathbf{F}$ - coronary angiogram showing connections between coronary arteries and tumor vessels, G, $\mathbf{H}$ - follow-up echocardiography and CT scan

\section{Acknowledgments}

Andrzej Surdacki and Dariusz Dudek are joint senior authors of this work.

\section{Conflict of interest}

The authors declare no conflict of interest.

\section{References}

1. Shepard MB, Mohiaddin R. Tumors of the heart. Future Cardiol 2010; 6: 181-93.

2. Joseph AL, Wilkow FE, Olivier JJ, Joseph GJ, Glancy DL. Angiosarcoma of the heart presenting as subacute pericarditis. Am J Cardiol 2017; 119: 941-3.
3. Esposito A, De Cobelli F, Ironi G, et al. CMR in the assessment of cardiac masses: primary malignant tumors. JACC Cardiovasc Imaging 2014; 7: 1057-61.

4. Nensa F, Tezgah E, Poeppel TD, et al. Integrated 18f-fdg pet/ $\mathrm{mr}$ imagingin the assessment of cardiac masses: a pilot study. J Nucl Med 2015; 56: 255-60.

5. Leja MJ, Shah DJ, Reardon MJ. Primary cardiac tumors. Tex Heart Int J 2011; 38: 261-2. 